\title{
Surgical indications in patients with an intracerebral hemorrhage due to a ruptured middle cerebral artery aneurysm
}

\author{
Masami Shimoda, M.D., Shinri Oda, M.D., Yoshiaki Mamata, M.D., Ryuichi Tsugane, M.D., and \\ Osamu Sato, M.D.
}

The new information from this small retrospective study of middle cerebral artery aneurysms and intracerebral hemorrhage associated with their rupture is that the outcome is better if the clot is contained within brain parenchyma without spillage into the subarachnoid space, whether it is located only in the sylvian fissure or becomes diffuse. The other prognostic items identified, such as, clot size, neurological condition, age, and operative problems, are well described by others. The study presents a logical story and conclusion, albeit from a small and retrospective point of view. The problem presented by multiple treatment variables besides surgery remain inherent in this retrospective approach to the problem.

Click here to return to Article 3. 